\title{
Tuberculose esplênica durante tratamento de psoríase com infliximabe
}

\author{
Splenic tuberculosis during psoriasis treatment with infliximab
}

\author{
Gleyce Tavares de Melo Fortaleza ${ }^{1}$ \\ Josemir Belo dos Santos ${ }^{3}$ \\ Perla Gomes ${ }^{5}$
}

\author{
Maria de Fátima de Medeiros Brito \\ Ana Roberta Figueiredo ${ }^{4}$
}

Resumo: A psoríase é doença crônica que afeta a pele e, eventualmente, as articulações. A terapia biológica age bloqueando citocinas inflamatórias implicadas na patogênese da doença, dentre elas o fator de necrose tumoral alfa. Este também tem papel na defesa do hospedeiro contra o Mycobacterium tuberculosis. Relata-se um caso de psoríase com boa resposta ao infliximabe, porém com desenvolvimento de tuberculose esplênica durante o tratamento.

Palavras-chave: Psoríase; Terapia biológica; Tuberculose

\begin{abstract}
Psoriasis is a chronic inflammatory disease affecting the skin and occasionally the joints. The biological agents have been engineered to target a specific step in the inflammatory cascade that leads to psoriasis, including tumor necrosis factor, which has a central role in the host defense against Mycobacterium tuberculosis. This is a case report about a psoriatic patient who presented splenic tuberculosis during treatment with infliximab.
\end{abstract}

Keywords: Psoriasis; Biological therapy; Tuberculosis

\section{INTRODUÇÃO}

A psoríase é doença crônica de curso intermitente que afeta a pele e, em alguns casos, as articulações. A gravidade da doença é bastante variável, sendo que em suas formas mais graves há grande comprometimento da qualidade de vida dos pacientes. Até o momento não há cura definitiva para a psoríase, sendo objetivos do tratamento o rápido controle da doença e a remissão prolongada.
Formas leves podem ser tratadas com agentes tópicos, enquanto formas moderadas e graves geralmente necessitam de fototerapia ou tratamento sistêmico, incluindo medicações como metotrexato, acitretina, ciclosporina ou agentes biológicos. ${ }^{1}$

Os agentes biológicos representam grande aquisição no arsenal terapêutico da psoríase. Essas drogas foram desenvolvidas para atingir alvos especí- 
ficos na cascata inflamatória que leva à doença. Podem ser subcategorizados como inibidores de células $\mathrm{T}$ ou antagonistas de citocinas. ${ }^{2,3}$ Entre os antagonistas de citocinas, inclui-se o infliximabe, um inibidor do fator de necrose tumoral alfa (TNF- $\alpha$ ). Embora a terapia biológica seja considerada segura e eficaz, é importante estar alerta para potenciais riscos associados ao seu uso.

\section{RELATO DO CASO}

Homem branco, de 43 anos, portador de psoríase há nove anos, sem acometimento articular. Ao exame, observavam-se placas eritêmato-descamativas no tronco, membros inferiores e couro cabeludo (Figuras 1 e 2). A doença não respondeu à terapêutica tópica e o paciente apresentou intolerância ao tratamento com acitretina e metotrexato, tendo sido indicado o uso do infliximabe. Havia indicação de fototerapia, porém não estava disponível no serviço. Exames laboratoriais iniciais foram todos normais, com teste tuberculínico anérgico (zero) e radiografia de tórax sem alterações. Foi iniciado, então, esquema de indução com infliximabe - infusão de $50 \mathrm{mg} / \mathrm{kg}$ de peso nas semanas zero, dois e seis -, associado ao uso de metotrexato, $5 \mathrm{mg} / \mathrm{semana}$ por via oral, no intuito de inibir a produção de anticorpos. O paciente evoluiu com melhora importante das lesões cutâneas (Figuras 3 e 4); porém, um mês após a terceira dose do infliximabe, passou a apresentar quadro de febre vespertina diária, astenia e perda de peso. Realizados hemograma, hemoculturas, sumário de urina, urocultura, radiografia de tórax e de seios da face, ultrassonografia de abdome e baciloscopias do escarro, que não evidenciaram alterações. Tomografia computadorizada de tórax mostrou derrame pleural à esquerda, associado a atelectasia passiva dos segmentos basais do lobo inferior, além de tênues opacidades nodulares do tipo vidro fosco no lobo superior direito (Figura 5). Estudo do líquido pleural mostrou padrão de exsudato, dosagem de adenosina deaminase $=34$ U/L e demais parâmetros inespecíficos. A tomografia computadorizada de abdome evidenciou aumento de volume do baço, que apresentava aspecto heterogêneo, com múltiplas e pequenas imagens isoatenuantes no parênquima, hipodensas na fase contrastada e de caráter inespecífico, além de intensificação da gordura mesentérica, com aspecto reticulado, sugestiva de acometimento inflamatório (Figura 6). Realizada punção esplênica com culturas para bactérias, fungos e bacilo de Koch (BK), sendo todas negativas. Reação em cadeia de polimerase para BK no sangue e na urina também foi negativa. Sorologias para paracoccidioidomicose e histoplasmose resultaram não reagentes. Exame histopatológico de fragmento esplênico mostrou esplenite granulomatosa de natureza não determinada. Colorações de Ziehl-Neelsen e PAS não revelaram bacilos álcool-ácido-resistentes ou fungos. Foi iniciado tratamento empírico com esquema I para tuberculose (rifampicina, isoniazida e pirazinamida), levando à regressão total dos sintomas e das lesões esplênicas. As lesões cutâneas da psoríase não reincidiram até o presente momento.

\section{DISCUSSÃO}

A psoríase é dermatose crônica de ocorrência universal, caracterizada pela presença de lesões eritêmato-escamosas. Na sua patogenia há aceleração do ciclo germinativo epidérmico, com marcante encurtamento do tempo da renovação celular nas lesões. É

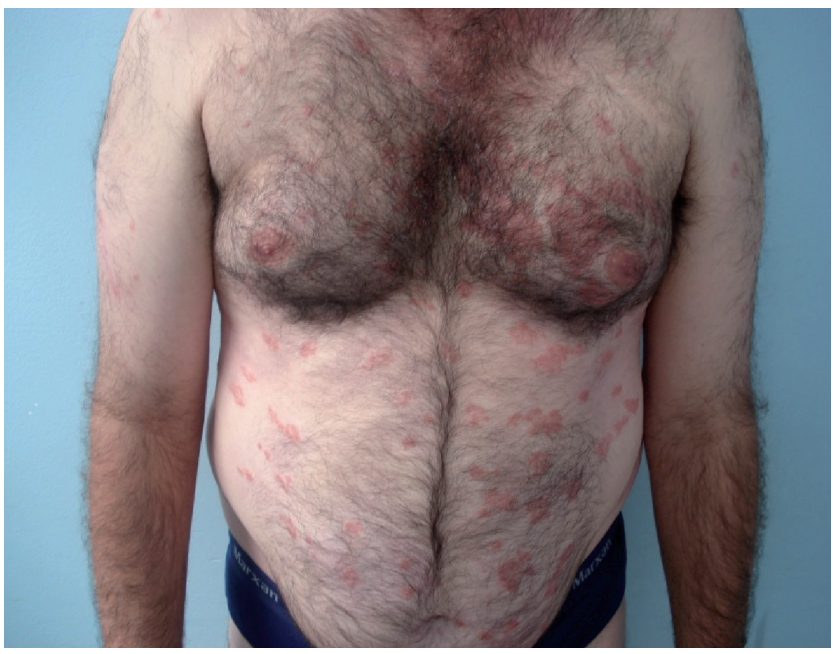

Figura 1: Placas eritêmato-descamativas no tronco, antes do início do tratamento com infliximabe

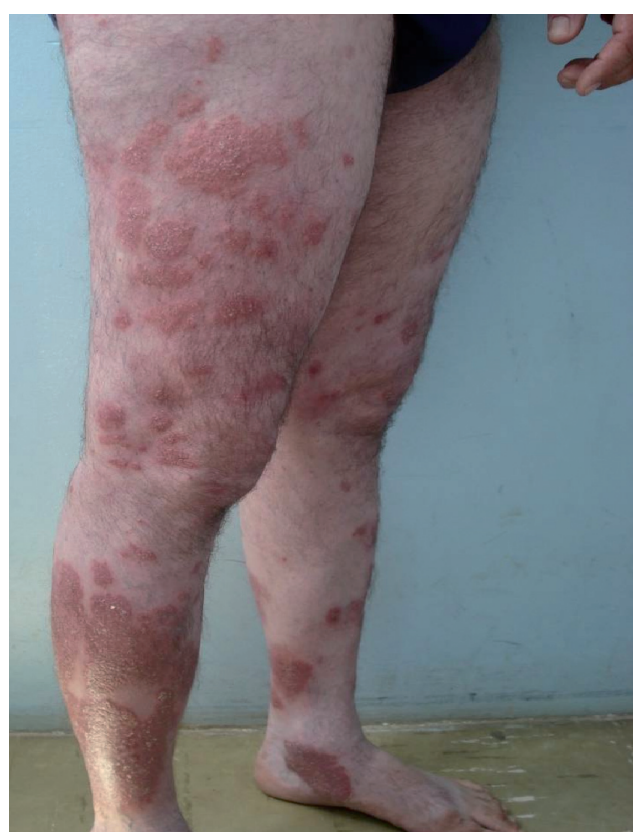

Figura 2:

Placas eritêmatodescamativas nos membros inferiores, antes do início do tratamento com infliximabe 


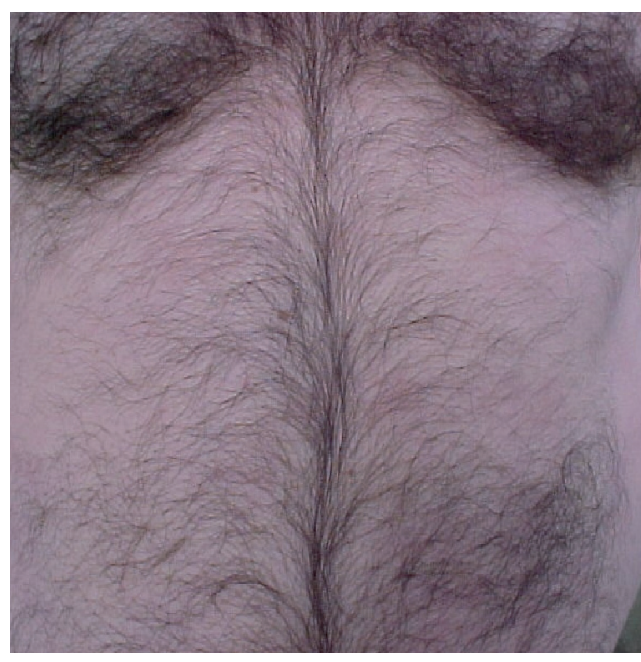

Figura 3 :

Regressão das lesões de psoríase no tronco, na semana 6 do tratamento com infliximabe

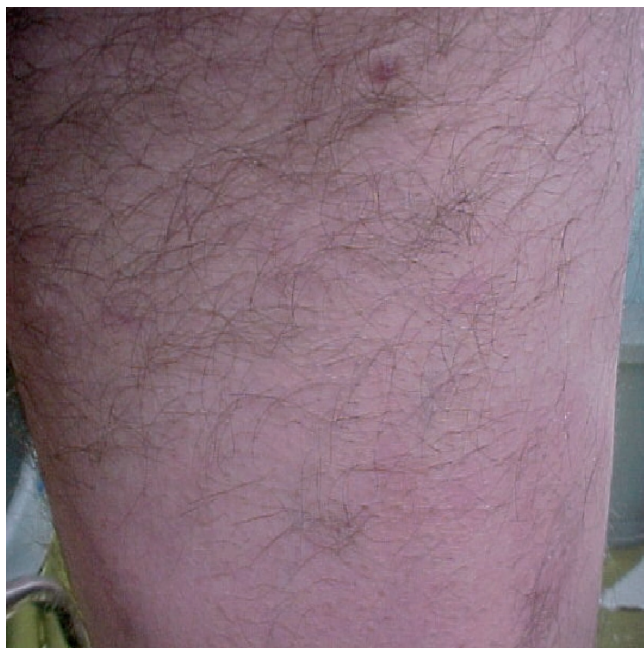

Figura 4 Regressão das lesões de psoríase no membro inferior direito, na semana 6 do tratamento com infliximabe

condição mediada por células $\mathrm{T}$ ativadas, exibindo padrão de citocinas do tipo Th1, especialmente interleucina 2 e TNF- $\alpha{ }^{4}$

Estudos de qualidade de vida na psoríase revelam um impacto semelhante ao visto nos casos de câncer, cardiopatias e artrite. $^{5}$ Pacientes com doença grave constituem aproximadamente $20 \%$ a $30 \%$ dos casos e frequentemente necessitam de tratamento sistêmico. Todas as terapias sistêmicas para psoríase estão associadas a potencial toxicidade a longo prazo, além de elevado custo e possibilidade de insucesso. A terapia biológica engloba um grupo de drogas destinadas a bloquear etapas específicas da cascata inflamatória implicada na patogênese da psoríase. Atualmente, a terapia biológica compreende dois grupos: (1) agentes antagonistas do TNF- $\alpha$ (p. ex., infliximabe, etanercepte, adalimumabe) e (2) agentes contra as células $\mathrm{T}$ ou células apresentadoras de antígenos (p. ex., efalizumabe, alefacepte). ${ }^{6}$

$\mathrm{O}$ infliximabe é um anticorpo monoclonal quimérico que inibe competitivamente o TNF- $\alpha$ tanto

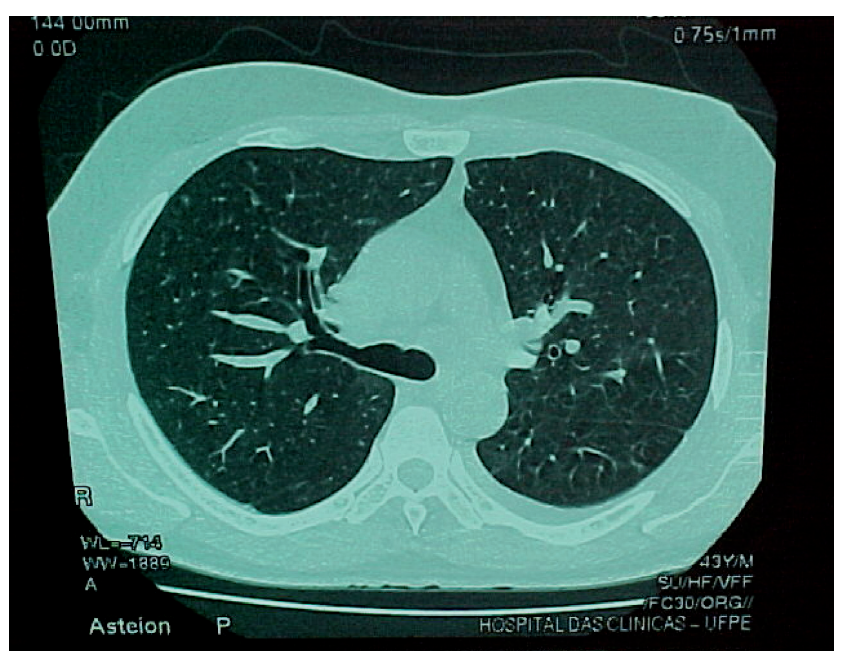

FIGURA 5: TC de tórax: derrame pleural à esquerda, associado a atelectasia passiva dos segmentos basais do lobo inferior esquerdo

solúvel quanto em formas ligadas às membranas celulares. É indicado no tratamento da psoríase em placas, artrite psoriásica, doença de Crohn, espondilite anquilosante e artrite reumatoide, sendo administrado por infusão endovenosa. Como se trata de um agente imunossupressor, a ocorrência de infecções é uma grande preocupação nos pacientes em uso de infliximabe, assim como de outros agentes anti-TNF- $\alpha$. Em nosso meio, há uma preocupação especial com a tuberculose (TB). ${ }^{1}$

O TNF- $\alpha$ é uma citocina pró-inflamatória produzida pelos linfócitos T, macrófagos e ceratinócitos, sendo encontrada em níveis elevados na pele dos portadores de psoríase. Ele estimula a produção de outras citocinas, incluindo a interleucina 1 (IL-1), IL-6 e IL-8, além de promover a proliferação de ceratinócitos.

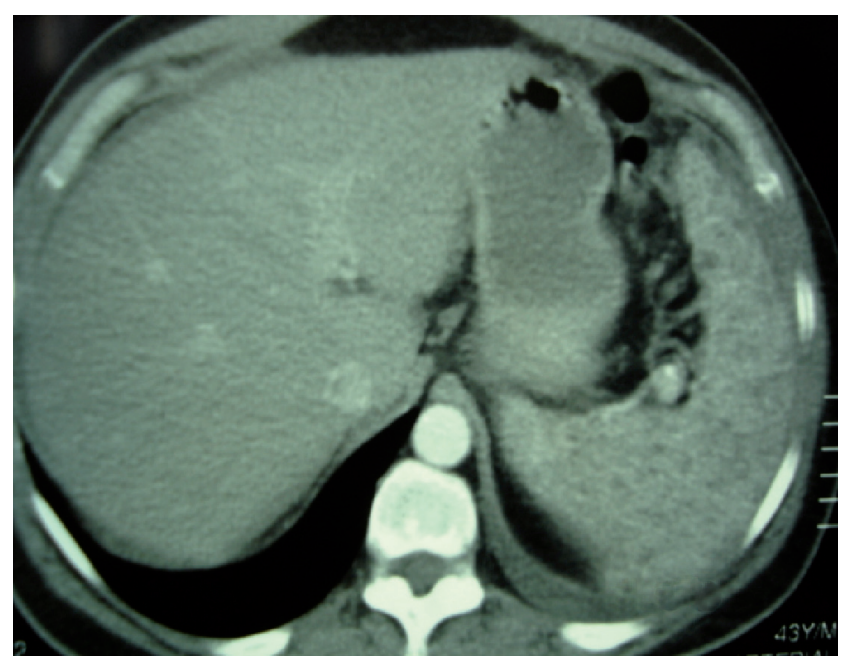

Figura 6: TC de abdome: baço aumentado de volume, com aspecto heterogêneo e múltiplas imagens isoatenuantes no parênquima 
Todos esses sinais podem levar à inflamação em vários órgãos, tais como pele e articulações. Com a neutralização dessa citocina pelas medicações anti-TNF- $\alpha$, há uma interrupção da cascata inflamatória essencial na fisiopatologia da psoríase e artrite psoriásica. ${ }^{7}$

Os efeitos do TNF- $\alpha$ não apenas são importantes nas desordens inflamatórias, mas também têm um papel crucial na defesa do hospedeiro contra o Mycobacterium tuberculosis. A resposta imune humana é altamente eficiente em conter a infecção primária resultante da exposição ao BK; entretanto, nem todos os micro-organismos viáveis são eliminados em alguns indivíduos. O bacilo é capaz de permanecer latente por períodos em que o indivíduo infectado é assintomático - condição conhecida como tuberculose latente -; porém, a qualquer momento pode desencadear a doença. ${ }^{8} \mathrm{O}$ TNF- $\alpha$ está envolvido na destruição dos bacilos pela ativação dos macrófagos e na prevenção da disseminação da infecção pelo estímulo à formação de granulomas. Uma vez que o TNF- $\alpha$ está implicado tanto na proteção contra a infecção pela micobactéria quanto na patogênese da tuberculose, não é surpresa que o uso dos anti-TNF- $\alpha$ esteja associado a aumento na incidência da TB. Embora alguns casos resultem de nova infecção pelo M. tuberculosis, a maioria resulta de reativação de TB latente. ${ }^{10}$ Além disso, apresentações atípicas da TB, como formas extrapulmonares e disseminadas, são mais frequentes durante o tratamento com qualquer um dos agentes anti-TNF- $\alpha .^{11}$

O Centro de Prevenção e Controle de Doenças (CDC) recomenda a realização de teste tuberculínico em todos os pacientes que serão tratados com inibidores do TNF- $\alpha .{ }^{12}$ Induração igual ou superior a $5 \mathrm{~mm}$ após 48 a 72 horas é considerada positiva para qualquer paciente candidato ao uso dos anti-TNF- $\alpha$. Devese atentar para o fato de que indivíduos imunocomprometidos podem ser anérgicos ao teste tuberculínico; consequentemente, uma induração inferior a 5 mm não deve excluir definitivamente a possibilidade de infecção pelo M. tuberculosis. Embora apresente efeito imunossupressor, o tratamento com anti-TNF não altera a resposta ao teste tuberculínico. ${ }^{11}$

A positividade do teste tuberculínico pode significar infecção latente pelo $M$. tuberculosis ou doença ativa. O raio-X de tórax é um importante teste de triagem para tentar diferenciar as duas condições e deve ser realizado em todos os pacientes com teste tuberculínico positivo em programação de tratamento com biológicos. ${ }^{11}$ Achados suspeitos ao raio-X devem levar à progressão da investigação com teste de escarro ou outros exames que forem necessários. Confirmado o diagnóstico de $\mathrm{TB}$, tratamento com esquema tríplice deve ser instituído. Indivíduos com teste tuberculínico positivo, porém sem achados radiológicos sugestivos de $\mathrm{TB}$, devem ser considerados portadores de TB latente e receber tratamento profilático antes do início da terapia com os agentes anti-TNF. ${ }^{13}$

O tratamento da TB latente mostrou-se eficiente em prevenir o desenvolvimento de TB ativa em $60 \%$ a $70 \%$ dos pacientes, porém mesmo com boa aderência ao tratamento profilático alguns casos de TB podem ocorrer. ${ }^{14}$ Além da profilaxia, é de suma importância manter vigilância para os casos de TB ativa e para manifestações atípicas de tuberculose em todos os pacientes em uso de agentes anti-TNF- $\alpha$. Nesses casos, a terapia biológica deve ser suspensa, iniciando-se tratamento com esquema tríplice. ${ }^{13}$

A terapia biológica demonstrou ser efetiva no tratamento da psoríase. Ela representa uma opção terapêutica efetiva para pacientes que não toleram o tratamento convencional ou para aqueles em que este não foi suficiente. Entretanto, como o TNF- $\alpha$ é também uma citocina importante no combate a infecções, particularmente à tuberculose, o uso de drogas antiTNF- $\alpha$ está associado a aumento do risco de desenvolvimento de TB ativa. 


\section{REFERÊNCIAS}

1. Zeichner JA, Lebwohl M. Potential complications associated with the use of biologic agents for psoriasis. Dermatol Clin. 2007;25:207-13.

2. Weinberg JM, Saini R, Tutrone WD. Biological therapy for psoriasis - the first wave: infliximab, etanercept, efalizumab and alefacept. J Drugs Dermatol. 2002;1:303-10.

3. Kazlow Stern D, Tripp JM, Ho VC, Lebwohl M. The use of systemic immune moderators in dermatology: an update. Dermatol Clin. 2005;23:259-300.

4. Menter A, Gottlieb A, Feldman SR, Van Voorhees AS, Leonardi CL, Gordon KB, et al. Guidelines of care for the management of psoriasis and psoriatic arthritis: Section 1. Overview of psoriasis and guidelines of care for the treatment of psoriasis with biologics. J Am Acad Dermatol. 2008;58:826-50.

5. Sampogna F, Sera F, Abeni D, IDI Multipurpose Psoriasis Research on Vital Experiences (IMPROVE) Investigators. Measures of clinical severity, quality of life, and psychological distress in patients with psoriasis: a cluster analysis. J Invest Dermatol. 2004;122:602-7.

6. Smith $\mathrm{CH}$, Anstey AV, Barker JN, Burden AD, Chalmers RJ, Chandler D, et al. British Association of Dermatologists guidelines for use of biological interventions in psoriasis 2005. Br J Dermatol. 2005; 153:486-97.

7. Gottlieb AB. Infliximab for psoriasis. J Am Acad Dermatol. 2003;49(Suppl2):S112-7.

8. Targeted tuberculin testing and treatment of latent tuberculosis infection. This official statement of the American Thoracic Society was adopted by the ATS Board of Directors, July 1999. This is a Joint Statement of the American Thoracic Society (ATS) and the Centers for Disease Control and Prevention (CDC). This statement was endorsed by the Council of the Infectious Diseases Society of America. (IDSA), September 1999, and the sections of this statement. Am J Respir Crit Care Med. 2000;161:S221-47.
9. Ellerin $\mathrm{T}$, Rubin $\mathrm{RH}$, Weinblatt ME. Infections and anti-tumor necrosis factor alpha therapy. Arthritis Rheum. 2003; 48:3013-22.

10. Gardam MA, Keystone EC, Menzies R, Manners S, Skamene E, Long R, et al. Anti-tumour necrosis factor agents and tuberculosis risk: mechanisms of action and clinical management. Lancet Infect Dis. 2003;3:148-55.

11. Doherty SD, Van Voorhees A, Lebwohl MG, Korman NJ, Young MS, Hsu S, et al. National Psoriasis Foundation consensus statement on screening for latent tuberculosis infection in patients with psoriasis treated with systemic and biologic agents. J Am Acad Dermatol. 2008;59:209-17.

12. Guide for primary health care providers: targeted tuberculin testing and treatment of latent tuberculosis infection. [cited 2008 Jan 2]. Available from: www.cdc.gov/tb/pubs/LTBI/pdf/TargetedLTBI05.pdf.

13. Winthrop KL. Update on tuberculosis and other opportunistic infections associated with drugs blocking tumour necrosis factor (alpha). Ann Rheum Dis. 2005;64(Suppl4):iv29-30.

14. Carmona L, Gómez-Reino JJ, Rodríguez-Valverde V, Montero D, Pascual-Gómez E, Mola EM, et al. Effectiveness of recommendations to prevent reactivation of latent tuberculosis infection in patients treated with tumor necrosis factor antagonists. Arthritis Rheum. 2005;52:1766-72.

ENDEREÇO PARA CORRESPONDÊNCIA / MAILING ADDRESS: Gleyce Tavares de Melo Fortaleza Rua dos Navegantes, $n^{\circ}$ 541, ap. 301, Boa Viagem, 51021010 Recife $P E$

Tel./Fax: 81 3326-6304/81 9212-6702/81 8818-0101 E-mail: glefortaleza@botmail.com

Como citar este artigo/How to cite this article: Fortaleza GTM, Brito MFM, Santos JB, Figueiredo AR, Gomes P. Tuberculose esplênica durante tratamento de psoríase com infliximabe. An Bras Dermatol. 2009;84(4):420-4. 